2 Jarcho L W, Fred H L, Castle C H. Encephalitis and poliomyelitis in the adult due to Coxsackie virus group B, type 5. N Engl J Med 1963; 268: 235-8.

3 Hopkins I J. A new syndrome: poliomyelitis-like illness associated with acute asthma in childhood. Aust Paediatr $J$ $1974 ; 10: 273-6$.

4 Danta G. Electrophysiological study of amyotrophy associated with acute asthma (asthmatic amyotrophy). J Neurol Neurosurg Psychiatry 1975; 38: 1016-21.

5 Ilett S J, Pugh R J, Smithells R W. Poliomyelitis-likeillness after acute asthma. Arch Dis Child 1977; 52: 738-40.

Correspondence to Dr H K:son Blomquist, Department of Paediatrics, University Hospital, S-901 85 Umeå, Sweden.

\title{
An explanation for failure of impedance apnoea alarm systems
}

\author{
DAVID P SOUTHALL, JEAN M RICHARDS, K C LAU, AND ELLIOT A SHINEBOURNE \\ Department of Paediatrics, Brompton Hospital, and Cardiothoracic Institute, London
}

SUMMARY 24-hour recordings of the ECG and respiration, the latter from an impedance technique, have shown a phenomenon which could account for hitherto unexplained failures of impedance apnoea alarm systems. Whenever apnoea is accompanied by bradycardia there is a pronounced increase in the amplitude of the cardiac impulse on the respiration carrier. This imitates the respiration signal and prevents the alarm from sounding. Conversely, apnoea unaccompanied by bradycardia does not present this problem and is detected by the alarm. If impedance alarm systems are to be used to detect apnoea they must be accompanied by a heart rate (ECG) detector.

Impedance alarm systems are widely used for the detection of apnoea in the newborn infant. They are often used in the routine care of preterm infants, 25 to $84 \%$ of whom are likely to have apnoeic attacks. ${ }^{1-2}$ They have also been advocated for the protection of those infants at special risk of sudden infant death. ${ }^{3-4}$ Failures of impedance apnoea alarm systems, however, are known to occur and some have been reported. ${ }^{35}$ These failures have occurred with no detectable electrical fault in the monitoring system, and with effective electrode contact. We are currently using combined 24-hour electrocardiogram and respiratory tape recordings to study infants with apnoeic attacks. During this study a phenomenon was observed which could explain failures in impedance apnoea alarm systems.

\section{Methods}

Combined 24-hour tape recordings of ECG and respiration* were made from 4 pregelled electrodes. The respiration signal, from a separate portable impedance unit, was recorded from 2 electrodes

* Oxford Medical Systems. placed $10 \mathrm{~cm}$ apart on one side of the chest wall, and the ECG signal from 2 electrodes positioned over the heart on the opposite side of the chest. The tapes were analysed by a newly developed system* programmed to detect both variations in heart rate and rhythm from preset R-R interval limits on the ECG and apnoea of greater than 10 seconds' duration from the respiration channel.

\section{Patients and results}

Combined recordings of ECG and respiration were performed on 3 infants with apnoeic attacks. A phenomenon was consistently documented in these infants, and in many others, which probably explains some of the failures of impedance apnoea alarm systems.

Case 1. This baby boy was admitted to hospital at age 3 weeks with episodes of pallor, cyanosis, and apnoea. He was term and weighed $2 \cdot 36 \mathrm{~kg}$ at birth. He was monitored on an impedance alarm system (Hewlett Packard 78202B) set to trigger after more than 15 seconds of apnoea. A combined 24-hour ECG and respiration recording showed four episodes of apnoea (10-40 seconds in duration) with bradycardia of down to $66 / \mathrm{min}$. The apnoea alarm failed to sound with any of these episodes and the 24-hour recording showed that during all episodes the size of the cardiac impulse during bradycardia increased, resembling the respiration signal (Fig. 1).

Case 2. This baby girl, who weighed $1.60 \mathrm{~kg}$ and was born at 31 weeks' gestation, presented at age 5 weeks with apnoeic episodes. She was monitored on a combined heart rate (from ECG) and apnoea (from impedance pneumograph) alarm system (Hewlett Packard 78213D cardiorespirograph). The alarms were set to trigger for heart rates below $100 / \mathrm{min}$ 


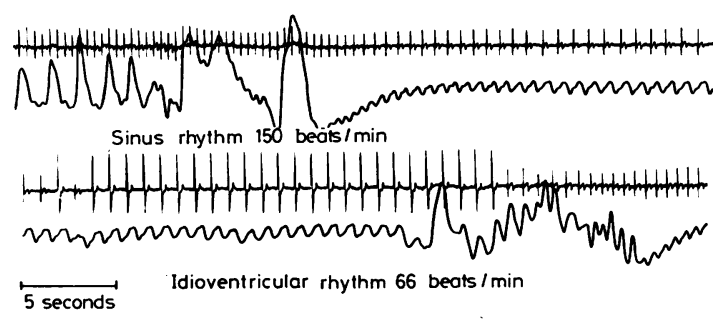

Fig. 1 24-hour ECG and respiration recording showing the ECG trace above a simultaneous respiration signal.

The initial heart rate is $150 / \mathrm{min}$. 15 seconds after the onset of recording there is an apnoeic episode lasting 40 seconds. 4 seconds after the onset of this episode there is a junctional escape rhythm which gradually becomes slower until 23 seconds after the onset of the apnoea, when an idioventricular rhythm of 66/min occurs. This rhythm persists until the onset of normal respiration.

When the heart rate falls the cardiac impression on the respiration trace becomes larger in amplitude and by the time of established junctional rhythm the cardiac impression is similar in volume to a low volume respiration signal.

This apnoeic episode failed to activate the impedance alarm system.

or for apnoeic episodes of more than 15 seconds. The combined 24-hour ECG and respiration recording showed 6 episodes of apnoea (10 to 60 seconds' duration) accompanied by bradycardia of as low as $60 / \mathrm{min}$ (Fig. 2). Unlike Case 1, during all episodes of apnoea and bradycardia on the 24-hour tape an alarm was activated.

Case 3. This baby girl weighed $1 \cdot 26 \mathrm{~kg}$ and was born at 28 weeks' gestation. Frequent apnoeic attacks, associated with bradycardias down to $60 /$ min were present. She was studied by 24 -hour recording at age 17 days while attached to an impedance alarm system (Hewlett Packard 78202B) set to trigger after 15 seconds of apnoea. During the 24 hours of recording a note was made of the time of alarms, and the duration of the apnoeic episode was estimated by adding 15 seconds to the length of time that apnoea persisted after the alarm had first sounded. The 24-hour tape was analysed without knowledge of the times of the apnoea alarms. During the recording the alarm sounded for four episodes of apnoea (15-20 seconds in duration) which in one case was associated with bradycardia of $96 / \mathrm{min}$. Four episodes of apnoea of between 15 and 32 seconds, all with bradycardias of between 66 and $82 / \mathrm{min}$, however, failed to be detected by the alarm system. In all of these latter episodes the size of the cardiac impulse during bradycardia increased in size and resembled the respiration signal.
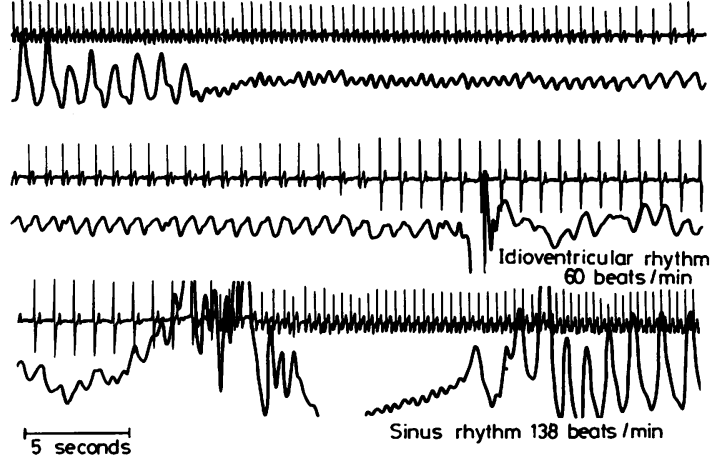

Fig. 2 24-hourECG and respiration recording showing the ECG trace above a simultaneous respiration signal.

The initial heart rate is $136 / \mathrm{min} .8$ seconds after the onset of the recording there is an apnoeic episode lasting 47 seconds. 20 seconds after the onset of the apnoea sinus bradycardia with a junctional escape rhythm begins. 20 seconds later there is an idioventricular escape rhythm of $60 /$ min which lasts for 7 seconds before a large breath occurs. A further apnoeic episode of 18 seconds follows this breath and an idioventricular rhythm continues during this apnoea until artificial ventilation with $a$ bag and face mask is started. Almost immediately the heart rate increases and the ECG reverts to sinus rhythm.

When the heart rate falls the cardiac impression on the respiration trace becomes larger in amplitude. By the time of established junctional rhythm the cardiac impression resembles a shallow respiration signal.

\section{Discussion}

The technique of impedance pneumography used to produce the 24-hour recording of respiration is identical with the method used by impedance apnoea alarm systems.

When apnoea is accompanied by bradycardia, if cardiac output is to be maintained, stroke volume has to increase. Concomitant with this there will be a greater difference in the volume of blood in the ventricles between systole and diastole and hence a greater difference in electrical impedance of the chest. The impedance changes between systole and diastole during slow heart rates thus may produce a signal in the respiratory carrier of comparable magnitude to that produced by shallow respiration. Thus if bradycardia accompanies apnoea, an apnoea alarm system may fail if it is dependent solely on an absence of the respiratory signal.

We suggest that in Case 1 this was the reason for failure of the apnoea alarm. The 24-hour tape recording in Case 3 confirmed that when apnoea was not accompanied by bradycardia the alarm system worked, but in apnoeic attacks where heart rate fell 
to below $85 / \mathrm{min}$ the alarm failed. The recordings also clearly demonstrate that alarm failures were due to the cardiac signal imitating the respiratory signal.

Prolonged apnoeic attacks in preterm infants may not only lead to death but can also result in hypoxaemia and brain damage. ${ }^{6}$ If intervention during an apnoeic attack is largely dependent on activation of an alarm, unreliable systems may do harm by creating a false sense of security.

A recent investigation describing infants with episodes of 'near-miss' sudden infant death looked after by their parents at home on impedance apnoea systems reported that 3 had died suddenly without their parents hearing the alarm. ${ }^{3}$ It is equally possible that because of the effects of associated bradycardia the alarm failed to sound.

Warburton et al. ${ }^{7}$ described the influence of the heart on the respiratory signal at normal heart rates in their studies in babies of artificially-induced upper airways obstruction. They did not, however, report the increase in amplitude of the cardiac signal with bradycardia. Most attacks of apnoea in preterm infants, however, are probably not due to the obstruction of the airways but to failure of the initiation of respiration. Such episodes are often accompanied by bradycardia which begins soon after, or simultaneously with, the onset of apnoea. ${ }^{18-9}$

We did not examine, in this study, the effects of different electrode positions on the size of the cardiac impulse. However, despite the respiration electrodes being placed on the opposite side of the chest to the heart during 24-hour recordings, there was still a pronounced increase in size of the cardiac signal when bradycardia occurred. It is likely that these changes could have been more prominent with electrodes positioned over the heart and therefore when monitoring for apnoea with an impedance alarm system it may be wise to position the electrodes as far as possible from the heart.

When will apnoea impedance alarms be activated if there is associated bradycardia? The answer to this appears to be, only if the heart rate falls below the lower level set for respiratory rate (that is $<6 / \mathrm{min}$ if the alarm is set to trigger after a 10 -second delay) or if stroke volume is so small due to depressed cardiac output that it does not produce a significant respiratory signal. At this stage it may be too late to resuscitate the infant.

In Case 2 there were no failures of the alarm system but in this instance the alarm might have been activated either by apnoea or by bradycardia. In our opinion it was the bradycardia or prolongation of R-R interval on the ECG, not apnoea, that triggered the alarm.
The implications of these findings are important as the use of apnoea impedance alarm systems is widespread. If these alarms are to be used it would appear necessary to add some form of heart rate detector (such as ECG) to the impedance system. It may be insufficient to monitor ECG alone because occasionally the heart rate does not fall to below $80 /$ min until more than 40 seconds of apnoea has occurred, as reported by Daily et al. ${ }^{1}$ Their study also showed that if peripheral stimulation is delayed until 30 seconds after the onset of apnoea, 8\% (43 out of 540) of attacks cannot be terminated by stimulation alone and require positive pressure ventilation.

We thank Dr R Dinwiddie and Dr D Mathew for referring the infants for study, the Oxford Instrument Company for their assistance in developing the 24-hour recorder, and Elaine Fowler and Dorothy Lewis for their help in preparing the manuscript.

D P S is supported by a grant from the Board of Governors of the Brompton and National Heart and Chest Hospitals, and J M R by a grant from the Foundation for the Study of Sudden Infant Death.

\section{References}

1 Daily W J R, Klaus M, Meyer H B P. Apnea in premature infants: monitoring, incidence, heart rate changes, and an effect of environmental temperature. Pediatrics $1969 ; 43$ : 510-18.

2 Alden E R, Mandelkorn T, Woodrum D E, Wennberg R P, Parks C R, Hodson A. Morbidity and mortality of infants weighing less than 1000 grams in an intensive care nursery. Pediatrics $1972 ; 50: 40-9$.

3 Kelly D H, Shannon D C, O'Connell K. Care of infants with near miss sudden infant death syndrome. Pediatrics 1978; 61 : 511-4.

4 Steinschneider A. A re-examination of "the apnea monitor business'. Pediatrics 1976; 58: 1-5.

5 Stein I M, Shannon D C. The pediatric pneumogram: a new method for detecting and quantitating apnea in infants. Pediatrics 1975; 55 : 599-603.

6 Bacola E, Behrle F C, de Schweinitz L, Miller H C, Mira M. Perinatal and environmental factors in late neurogenic sequela. I: Infants having birth weights under 1.500 grams, and II: Infants having birth weights from $1 \cdot 500$ to $2 \cdot 500$ grams. Am J Dis Child 1966; 112: 359-68; 369-74.

7 Warburton D, Stark A R, Taeusch H W. Apnea monitor failure in infants with upper airway obstruction. Pediatrics 1977; 60: 742-4.

8 Girling D J. Changes in heart rate, blood pressure, and pulse pressure during apnoeic attacks in newborn babies. Arch Dis Child 1972; 47: 405-10.

- Storrs C N. Cardiovascular effects of apnoea in preterm infants. Arch Dis Child 1977; 52: 534-40.

Correspondence to $\mathrm{Dr}$ D P Southall, Department of Paediatrics, Cardiothoracic Institute, Fulham Road, London SW3 6HP. 